\title{
Autonomous CONTROL SySTEM FOR A VEHICLE WiTH ACTIVELy ARTiculated Frame
}

\author{
Kharuzin Sergei Vadimovich, Ivanov Aleksandr Aleksandrovich \\ \& Shmakov Oleg Aleksandrovich
}
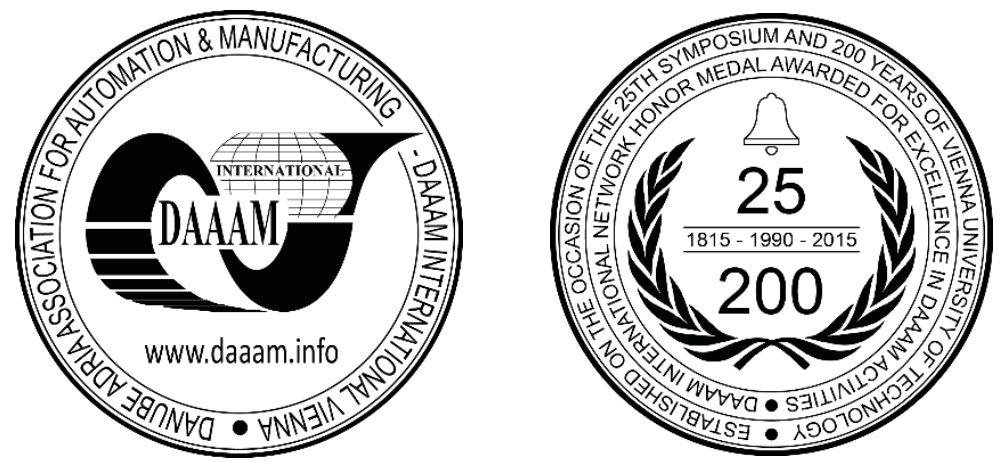

This Publication has to be referred as: Kharuzin, S[ergei]; Ivanov, A[lexander] \& Shmakov, O[leg] (2017). Autonomous Control System for a Vehicle with Actively Articulated Frame, Proceedings of the 28th DAAAM International Symposium, pp.0759-0766, B. Katalinic (Ed.), Published by DAAAM International, ISBN 978-3-90273411-2, ISSN 1726-9679, Vienna, Austria

DOI: $10.2507 / 28$ th.daaam.proceedings.107

\begin{abstract}
In this paper we suggest a structure of an autonomous control system for a six-wheeled vehicle with actively articulated frame that is traversing through cluttered spaces with undetermined placement of specified-type obstacles. Description of active frame transformation control strategies and algorithms also is given. Control algorithms utilize information that is provided by proprioceptive sensors (used in direct estimation of current vehicle configuration) and exterioceptive sensors (used in indirect estimation of the obstacle height and distance from it). These algorithms are active while vehicle overcomes random consequence of obstacles belonged to three classes: step (overcoming), step (descending) and ditch. For each obstacle type we suggest specific vehicle movement mode. Patterns of each feasible switching between modes and dependency between desired frame configuration and obstacle height are then discussed. Suggested strategies and algorithms are tested via complex virtual modelling of control system and vehicle dynamics.
\end{abstract}

Keywords: Unmanned ground vehicle; mobile robot; all-terrain vehicle; autonomous movement

\section{Introduction}

Unmanned ground vehicles that have actively articulated mechanisms as a part of their frames can effectively traverse through uneven terrain with randomly positioned obstacles. Height of an obstacle that can be overcome by these robots highly depends on reconfiguration manner and may exceed one of vehicle's nominal dimensions. However, adaptive reconfiguration or transformation of robot's frame under control of human operator that is observing both obstacle and robot is executable but challenging task. In real working conditions only visual information captured by robotic platform onboard cameras is provided to operator. Such information may be insufficient for effective control. In order to increase quality of control process, operator may be supplied with information captured by exterioceptive and proprioceptive sensors. But in some cases it can be not enough for efficient control of robotic platform. Moreover, complexity of incoming information paired with redundancy of robotic platform (for it has many degrees of freedom) can cause sensitive overload and decrease operator performance. Full or partial automation of the control process may be performed to establish robotic platform effective movement in unstructured environments. 
In this paper we suggest a structure of an autonomous control system for a six-wheeled vehicle with actively articulated frame that is traversing through cluttered spaces with undetermined placement of specified-type obstacles. Description of active frame transformation control strategies and algorithms also is given. Control algorithms utilize information that is provided by proprioceptive sensors (used in direct estimation of current vehicle configuration) and exterioceptive sensors (used in indirect estimation of the obstacle height and distance from it). These algorithms are active while vehicle overcomes random consequence of obstacles belonged to three classes: step (overcoming), step (descending) and ditch. For each obstacle type we suggest specific vehicle movement mode. Patterns of each feasible switching between modes and dependency between desired frame configuration and obstacle height are then discussed. Suggested strategies and algorithms are tested via complex virtual modelling of control system and vehicle dynamics.

\section{Problem statement}

In this paper we raise a problem of establishing robust control for articulated six-wheeled vehicle. Robotic platform is minimally equipped with exterioceptive sensors and features uncommon mechanical structure. Concerned device must efficiently traverse through cluttered spaces or move on uneven terrain. Furthermore, all platform active frame transformations must be performed in autonomous mode - without overriding from human-operator. This means that operator is only able to choose movement direction for platform. For method verification we use complex virtual model of robotic platform kinematics and dynamics.

\section{Related work}

The main part of research works in the area of developing obstacle negotiation algorithms for articulated devices with continuous ground trace of propulsion mechanisms was performed with wheeled and tracked robots.

While overcoming obstacles wheeled devices often rely on additional mechanisms and structural improvements. One of the common ways to increase passing ability of such robots is utilizing leg-wheel structures. The way of efficient usage of leg-wheel mechanisms in a role of adaptive suspension is presented in paper [1]. Each mechanism of a suggested fourwheeled robot consists of two linkages one of which is connected with robot's base and other with motorized wheel. Vertical loads and inclinometers are proposed by authors to be implemented as control algorithm inputs. Having been tested via virtual modelling this method has shown its efficiency. Another way to improve obstacle negotiation capability is to gather more information about robot's environment. Researchers [2] suggested torque control algorithms for minimizing wheel slip of the six-wheeled robot equipped with so-called tactile wheels and active suspension mechanism. Tactile wheels allow robot's computing unit to estimate direction and scale of wheel-terrain normal forces. Method has shown its efficiency with two different sets of tires used to achieve different friction force values. In paper [3] another approach was demonstrated. Authors have developed six-wheeled device with semi-passive compliant suspension mechanism. It consists of parallel linkages that hold side wheels and a front four-linked suspension front wheel. Rear wheel is fixed on the individual link. Experiments both with physical and virtual model of the robot have shown that it can overcome obstacles that is higher that device's wheel. Adaptive leg gait for four-wheeled robot with 5 actuated axes (4 for wheels and 1 for robot's frame twisting) has been shown in [4]. Considered adaptive semiautonomous movement pattern allows vehicle to negotiate singular step-like obstacles.

Another subject of discussion presented in recent scientific publications is developing situational control strategies for wheeled robots with common structures. Different strategies for overcoming and descending from step or moving over a ditch for six-wheeled vehicles were described in [5-7]. Fuzzy algorithm for obstacle classification under information gathered with range imaging device was developed in [8]. It allows robot to autonomously classify obstacles and choose desired movement pattern for their negotiation. Situational control and strategies for overcoming obstacles by six-wheeled robot was considered in [9]. Robot's computing unit monitors energy consumption in active joints that connect robots frame and wheel-legs. When consumption rises over preliminary stated threshold it considered that step-like obstacle has been met.

Some auxiliary methods suitable for many wheeled devices also were recently discussed by several authors. Wheel anti-slip method for robotic platform has been discussed in [10]. Main principles of this method may be used as recommendations for developing of effective anti-slip controller. Paper [11] describes tip-over algorithms for movement of wheeled vehicle on rough terrain.

Some studies have examined obstacle negotiation for tracked robots. Authors of [12] have presented algorithm for morphology control of the tracked vehicle with active tracked levers. Suggested algorithm utilizes information provided by an onboard camera and 3-axial gyroscope. The main issue of this work is that robot adapts to its environment without prior knowledge of the stair geometry. Paper [13] represents control system for tracked robot with same structure. Suggested control system was built based on fuzzy logic algorithms for control of track movement speeds. Another approach for establishing autonomous obstacle negotiation was presented in [14]. Authors have considered algorithms that utilize incomplete information about robot's environment gathered via probing its surroundings with sensible manipulator. Stages of development of wheel-tracked robot with reconfigurable tracks and control algorithms for such 
device have been discovered in [15]. This vehicle has unfordable tail that acts as a third footing for movement on flat terrain when both tracks are fully folded and act as wheel. In [16] two-phased motion algorithm for movement on ledges is suggested. The main issue of the proposed method is that it is not belong to the group of the classification-based methods. Therefore, robot desirable condition is generated based on the obstacle parameters.

We propose structure of control system that has reduced pre-requisites for robot's sensor equipment. The only exterioceptive information that is used by our system for task generation is information provided by optical range sensors and information about each vehicle's module orientation. Our approach is quite simple from the view of technical realization and can be implemented in already existing physical models without changing their mechanical structure. Suggested below strategies describes robotic platform behavior in three common scenarios reviewed by other authors: step (overcoming), step (descending) and ditch negotiation.

\section{Robotic platform}

3D CAD-model of articulated six-wheeled vehicle with 9 active drives is depicted at Fig. 1 (a). Robotic platform consists of 3 two-wheeled modules (pt. 1-3). Rotation axes of motorized wheels (pt. 4-9) are fixed to modules. Middle module is separated into two similar pieces connected via active rotational joint. It includes rotational drive that performs rotation of first and third modules in relation to middle module. The axis of such rotation is identical to middle motorized wheels axis. Achievable rotation angle belong to range of $\pm 90^{\circ}$. For their part, first and third modules each include one lateral and one rotational active joint. Lateral joint allows modules (pt. 1,2) to move towards and away from middle module (pt. 3). Rotational joint allows them to rotate around lateral movement axis. All considered active frame transformations performs under lateral drives movement and rotation of active joint that placed in middle module. Distance between axes of first and middle or third and middle modules may be expanded for up to $33 \%$ of nominal dimensions.

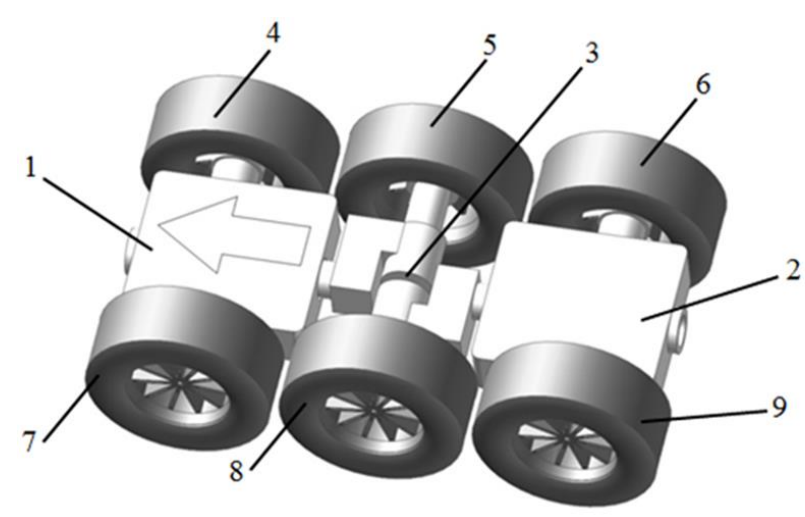

a)

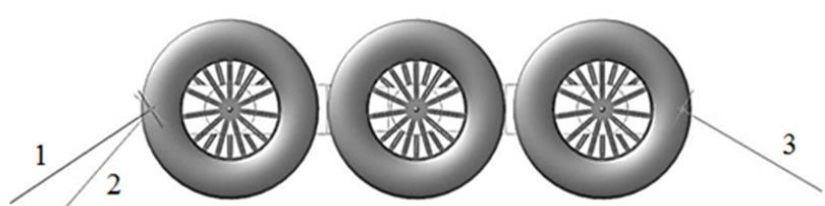

b)

Fig. 1. 3D CAD-model of transformable six-wheeled vehicle (a) and optical range sensor beams (b)

Information for obstacle discovery and classification is provided by three optical range sensors and 2 orientation sensors, each with two active axes. First two range sensors are placed on the frame of first module and one range sensor is placed on the rear module. Orientation sensors placed in both rear and front modules. Optical range sensors beams are depicted at Fig. 1 (b) (pt. 1) and (pt. 2-3). Angles between beams and horizontal plane are $30^{\circ}$ and $45^{\circ}$. With considered approach minimal number of proprioceptive sensors corresponds to amount of active drives. Number of external sensors, therefore, must be sufficient for obstacle classification and determination of vehicle condition.

\section{Structure of the control system}

Autonomous control system contains nodes as depicted at Fig. 2. Target generation node produces time-dependent expressions that characterize movement of active drives between update intervals. This process is based on discrete information that is periodically provided by exterioceptive/proprioceptive sensors and rules of obstacle classification. Regulator in this particular case plays standard role, it forms control signals based on error between target and current condition.

We suggest three obstacle classes: (1) obstacles that can be overcome by simple movement with passive rotation of all joints; (2) obstacles that can be overcome by performing a sequence of active frame transformations; (3) obstacles that can't be overcome in any affordable vehicle conditions and configurations. Rules for overcoming typical obstacles are realized for overcoming obstacles outlined in first two classes. Discovery of third class obstacles causes initiation of emergency stop algorithms. 


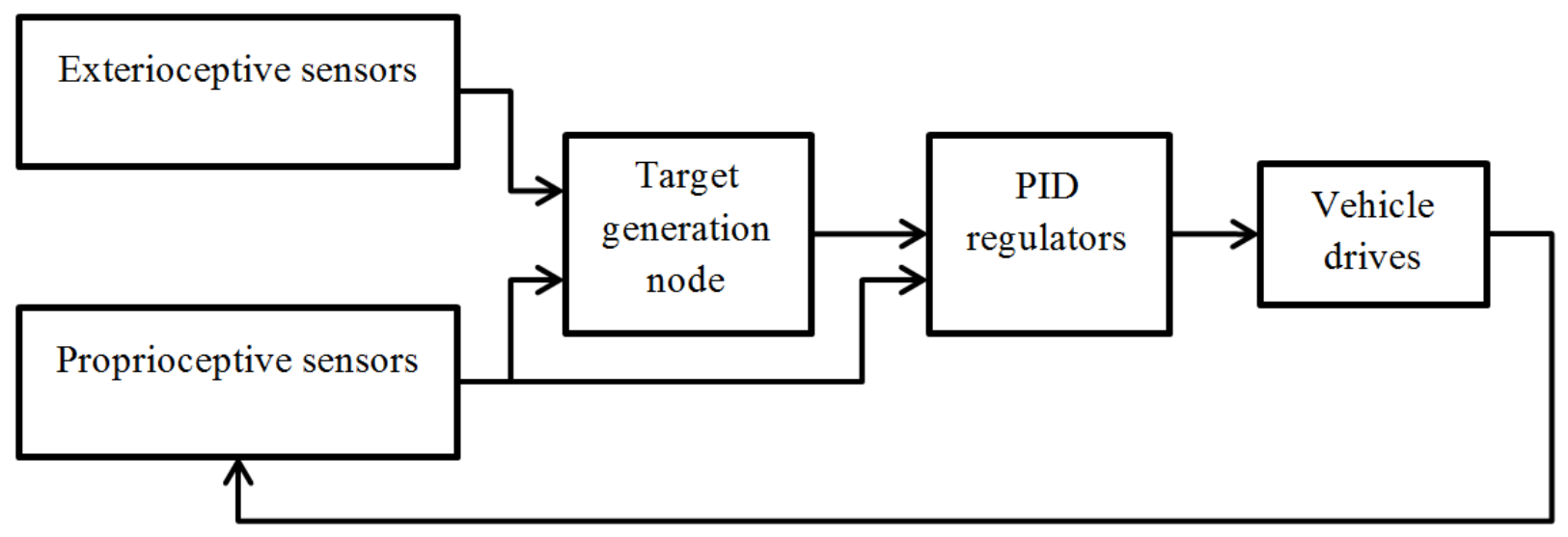

Fig. 2. Generalized structure of control system

\section{Strategies overview}

In current subsection we suggest behavioral strategies for five typical cases. These cases are: overcoming a step, descending from a step, ditch negotiation, simple forward movement and emergency stop in case of detection of obstacle that can't be overcome. Every typical case corresponds to specially designed movement mode.

Diagram of executable switches between modes is depicted at Fig. 3. It is possible to switch nominal mode (simple forward movement) to four different modes: overcoming a step, descending from a step, ditch negotiation mode and emergency stop mode. First three modes can be characterized by consequently executable stages of vehicle frame transformations. After finishing sequence of stages in each mode nominal mode become active.

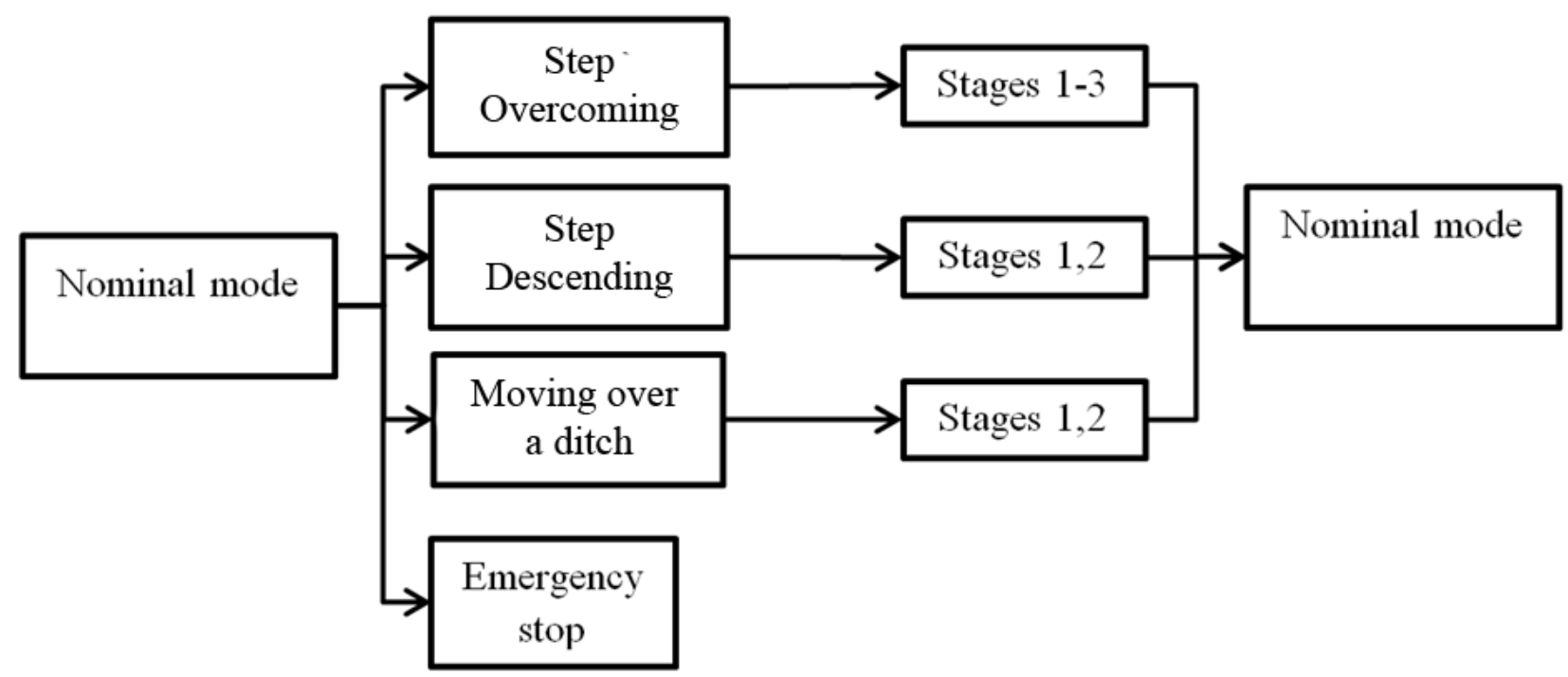

Fig. 3. Diagram of executable switches between movement modes

Obstacle discovery and classification performs under the values of estimated height of the obstacle and estimated distance to it. By estimated height we assume the length of vertical projection of interval point of between range sensor beam and obstacle surface intersection and point of front or middle wheels and terrain contact. In this particular case we assume that front and/or middle wheels are in contact with terrain.

For estimation of rear sensor group parameters we assume that rear and/or middle wheels are in contact with terrain. By estimated distance between vehicle and obstacle we assume horizontal projection of interval between beam origin and point where it intersects with an obstacle. Below we suggest expressions for calculating estimated parameters for range sensor that is placed on front module. Calculation diagram is depicted at Fig. 4. 


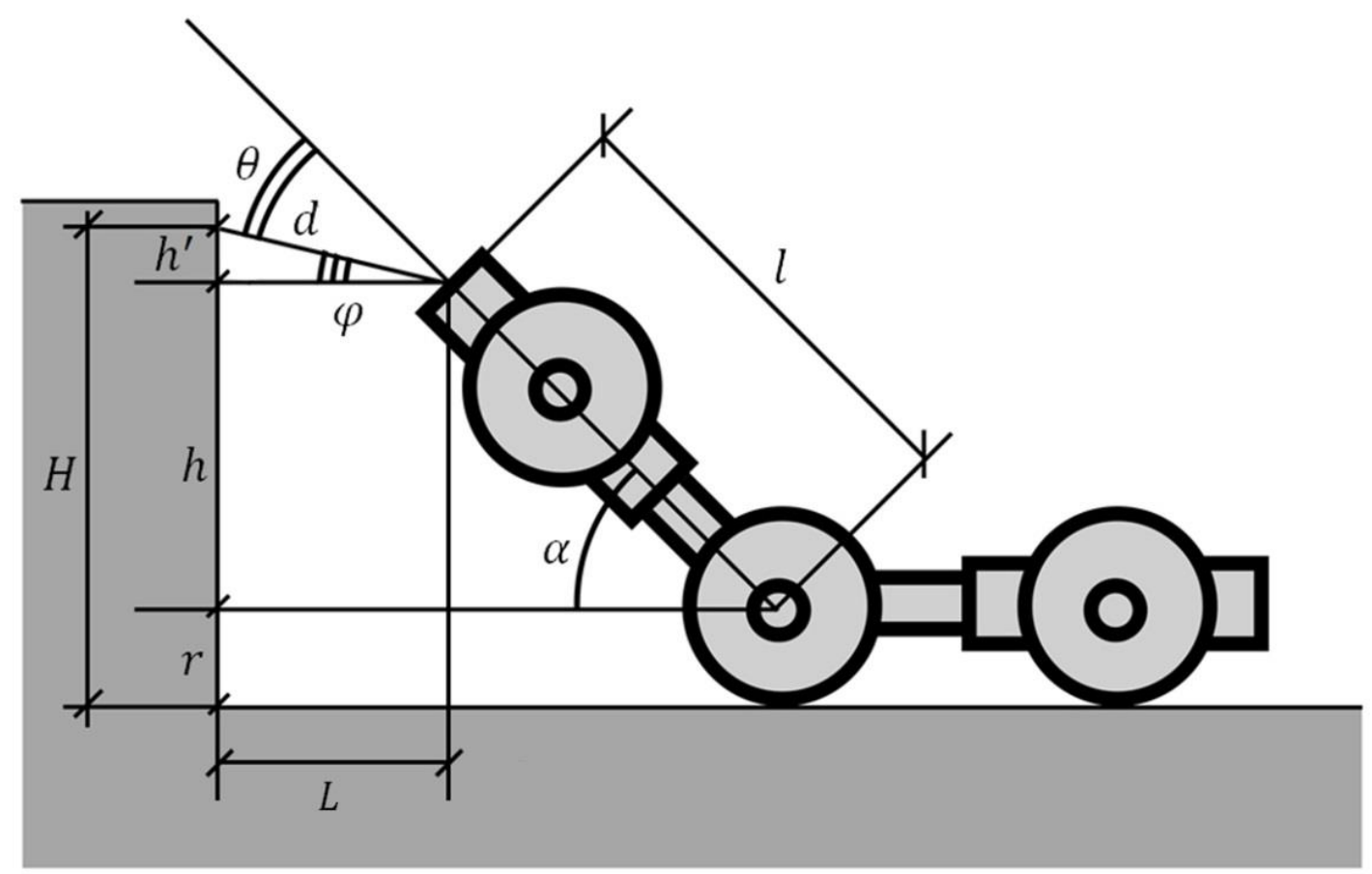

Fig. 4. Diagram for calculation of estimated parameters. Range sensor is placed on front module

Calculations of estimated height $H$ and distance $L$ are performed in accordance with equation (1) and (2):

$$
\begin{aligned}
& H=r+l * \sin (\alpha)+d * \sin (\alpha-\theta) \\
& L=d * \cos (\alpha-\theta)
\end{aligned}
$$

here $r$ is a wheel radius; $l$ - distance between front wheels axis and sensor beam starting point; $d$-interval between beam starting point and point of intersection between beam and obstacle; $\alpha$ - angle between rear and front modules; $\theta$ - angle that characterizes direction of sensor beam towards nominally horizontal plane.

First and second range sensors provide information for obstacle discovery and classification. If provided by second sensor estimated height corresponds to a cavity with depth that exceed wheel radius and provided by first range sensor estimated length is less or equal to maximum distance between platform adjacent pairs of wheels then ditch negotiation mode activates. If the ditch width exceeds distance between wheels of maximally exerted front module and wheels of middle module then mode of descending from escarp activates. Obstacle that classified as escarp corresponds to estimated height of second sensor that exceeds $1 / 4$ of wheel radius. This rule guarantees obstacle negotiation under a small dynamic friction. If estimated height of the obstacle exceeds preliminary established limits then emergency stop mode activates. If estimated height belong interval between $-\mathrm{r} / 2$ and $\mathrm{r} / 4$ then simple forward movement mode activates.

Movement of platform in environment with undetermined positions of typical obstacles can be represented as set of consequently carried stages that corresponds to movement modes. Simplified images of platform configurations at different stages of movement modes depicted at Fig. 5. If there are no obstacles on the way of platform then it moves in nominal mode (pt. 1). In this mode both front and rear modules are retracted and only rear pair of wheels is actuated. If nearest environment irregularity was classified as an escarp then nominal mode switches to the first stage of the escarp negotiation mode. Configuration of the platform when the first stage is finished depicted as (pt. 2). It corresponds to leap in estimated height and a length values due to range sensor beam begins to intersect with escarp floor. Configuration (pt. 3) can be achieved by rotating the front module in opposite direction with simultaneous correction of wheels speed. Finishing stage of escarp negotiation mode corresponds to (pt. 4). At this stage vehicle performs active frame transformation until its middle wheels get in contact with escarp floor. Then it lifts rear module and switches current mode to simple forward movement (pt. 5). Detection of a deep and sufficiently wide cavity leads to switching nominal mode to escarp descending mode (pt. 6). At this stage both front and rear modules extracts on the length that provides safe descend. This configuration corresponds to the case when simultaneous contact of frontal wheels and lower floor and middle wheels and upper floor is achievable. During this process only rear wheels are actuated. When achieving stage (pt. 8) through stage (pt. 7) front and middle wheels are actuated and PID regulator coefficients for middle transformation drive is set to be 10 times lower than its nominal condition. At the first stage of a ditch negotiation mode (pt. 9) front module extracts so distance between frontal and middle wheels become equal to ditch width. If range sensors have detected a floor the second stage (pt. 10) initiates. Stage consists of transferring middle module towards to the front module with simultaneous extraction of rear module (pt. 11). Platform current mode switches to nominal when rear range sensor detects floor. 

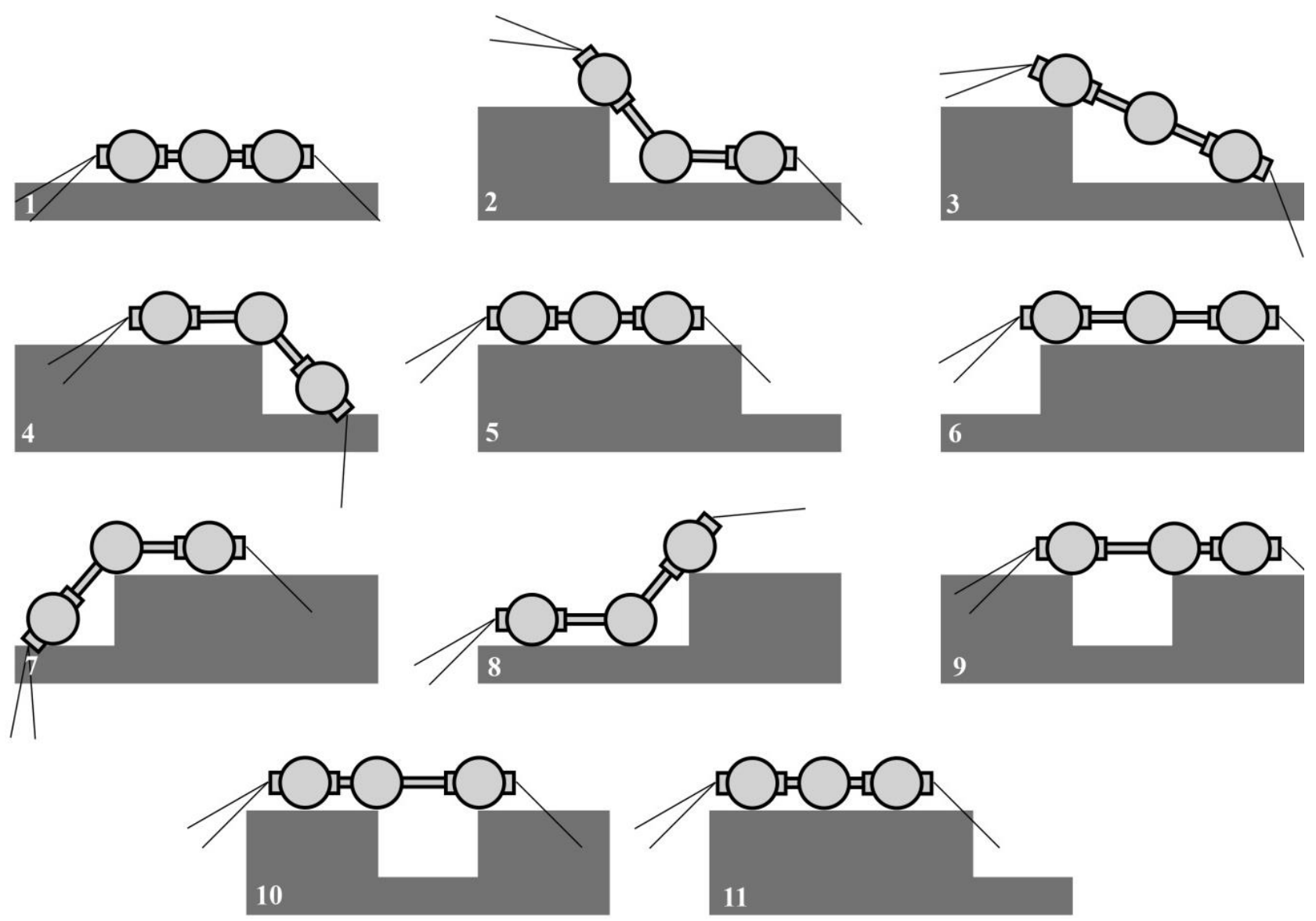

Fig. 5. Simplified images of different platform configurations at different stages of movement

\section{Control algorithm}

Control algorithm consists of 6 stages that are consequently executed on each virtual modelling step. In the first stage proprioceptive and exterioceptive information are gathered and obstacle height and distance to it are estimated. This information then is utilized to choose movement mode. Then based on current platform condition and current movement stage target drive conditions for robot transformation are generated. This target further is modified in correspondence to current targets for wheel rotation speeds and modules extraction. Equation (3) is suggested to be used for wheels speed correction in accordance to module extraction speed:

$$
\omega=\omega 0 \pm\left(v / r_{-} w\right)
$$

here $\omega$ is wheel speed; $v$ is module extraction speed; $r_{w}$ is wheel radius.

At the final stage errors between current and target platform configurations are calculated and send to PID regulators. Torques and forces that are formed this way then applied to mechanical parts of the actuators.

\section{Virtual experiment}

Suggested strategies and algorithm have been tested on complex virtual model of dynamics and kinematics for sixwheeled articulated platform. During virtual testing each step of the suggested algorithm was consequently performed with frequency of $100 \mathrm{~Hz}$. Torques and forces formed by control system model were applied to mechanical parts of platform actuators. Testing was performed under the various parameters of wheel and ground contacts. At each testing iteration we were selecting dynamic friction from the range of 0,3 to 0,7 .

We discuss algorithm efficiency under the example of step overcoming process. Dynamic friction coefficient during considered virtual test was chosen to be 0,3 . Obstacle negotiation process depicted at Fig. 6 . While approaching to the obstacle platform moves in first mode (pt. 1). Then lifting process of the first module begins (pt. 2). After achieving preliminary assigned angle platform start to rotate front module in opposite direction (pt.3, 4). At third stage rear module are lifted to get in contact with escarp floor (pt. 5). Platform returns in nominal mode at final stage (pt. 6). 


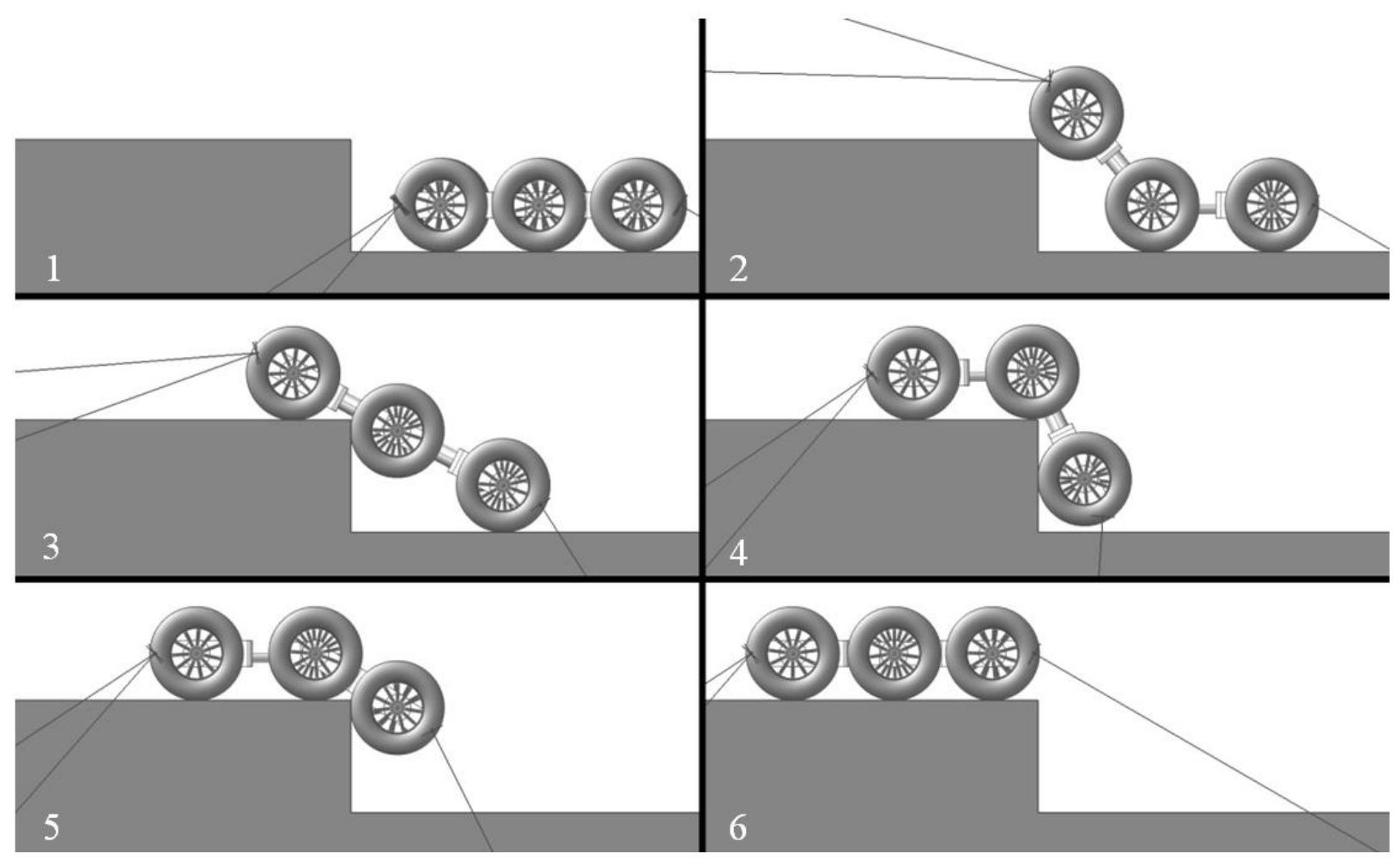

Fig. 6. Obstacle negotiation process

\section{Conclusion}

The approach for solving a problem of establishing robust control for articulated six-wheeled vehicle that is moving through cluttered spaces has been discussed. During concerned research new strategies for overcoming relatively large obstacles have been developed. Rules for obstacles classification were suggested. Continuously preformed platform transformation stages allows articulated vehicle to overcome a set of specified obstacles belonged to classes of step (overcoming), step (descending) and ditch. Preliminary efficiency approval of suggested control scheme and strategies was performed via complex virtual modelling of platform kinematics and dynamics. Virtual experiments showed that suggested strategies provide effective negotiation of specified obstacles. Including it in form of algorithms in the control system of transformable robotic platform provides it with capability to overcome relatively large obstacles with dynamic friction coefficient between wheels and ground higher than 0,3.

The main disadvantage of the suggested control system is that it has highly determined structure. Therefore switching between movements modes is not possible before exit condition for current mode is achieved. Furthermore described virtual modelling was performed with simplified models of idealized obstacles. For real world conditions additional rules for transformation and classification might need to be developed. Nevertheless we consider that stated above problem is solved. Based on achieved results we suggest that further implication of suggested algorithms will allow physical model of the platform to effectively traverse through undetermined environments with minimal aid of human-operator.

Future work is focused on further algorithm refining, extending the set of classified obstacles and expanding control scheme capabilities on the case of three-dimensional movement.

\section{Acknowledgements}

This research is supported by the Ministry of Education and Science of Russia (an agreement № 14.578.21.0124 on granting for the implementation of applied scientific research and experimental development). Unique identificator of ASRED - RFMEFI57815X0124.

\section{References}

[1] Alexey, B.; Dmitriy K. \& Eugeny, L. (2016). Use of walking mechanisms as an adaptive suspension of a planet rover, Proceedings of the 26th DAAAM International Symposium, ISSN 1726-9679, ISBN 978-3-902734-07-5, Katalinic, B. (Ed.), pp. 0989-0996, Published by DAAAM International, Vienna, Austria, DOI 10.2507/26th.daaam.proceedings.139

[2] Bin X.; Cedric P.; Ambroise K.; Roland S. \& Fuchun S. (2011). Composite control based on optimal torque control and adaptive kriging control for the crab rover, Proceedings of the 2011 IEEE International Conference on Robotics 
and Automation, Shanghai International Conference Center, ISSN 1050-4729, ISBN 978-1-61284-386-5, Published by IEEE, Shanghai, China, DOI 10.1109/ICRA.2011.5979800

[3] Min X.; Runhuai Y.; Yong C. \& Hongcheng X. (2011). Kinematics modeling and step climbing study of an allterrain wheeled mobile robot on uneven terrains, Proceedings of the 2011 International Conference on Electronic \& Mechanical Engineering and Information Technology, ISBN 978-1-61284-086-4, Published by IEEE, Harbin, China, DOI 10.1109/EMEIT.2011.6023597

[4] Shuro N. \& Kazuhisa I. (2012). Concept of adaptive gait for leg-wheel robot, RT-mover, Proceedings of the 2012 IEEE International Conference on Robotics and Biomimetics, ISBN 978-1-4673-2127-3, Published by IEEE, Guangzhou, China, DOI 10.1109/ROBIO.2012.6490982

[5] Kyeong B. L.; Sim J. K. \& Yong-San Y. (2010). Deliberative planner for ugv with actively articulated suspension to negotiate geometric obstacles by using centipede locomotion pattern, Proceedings of the International Conference on Control, Automation and Systems 2010, KINTEX, ISBN 978-89-93215-02-1, Published by IEEE, Gyeonggi-do, Korea, DOI 10.1109/ICCAS.2010.5670132

[6] Jingli L. \& Chunguang B. (2009). Study on the mobile robot reconfiguration control methods, Proceedings of the IEEE International Conference on Automation and Logistics, ISSN 2161-8151, ISBN 978-1-4244-4794-7, Published by IEEE, Shenyang, China, DOI 10.1109/ICAL.2009.5262605

[7] Weidong W.; Dongmei W.; Wei D.; Chun X. \& Pengfei S. (2012). The optimization of obstacle-crossing and the simulation in ADAMS of the composite six-wheeled-legged robot, Proceedings of the 2012 9th International Conference on Fuzzy Systems and Knowledge Discovery (FSKD 2012), ISBN 978-1-4673-0024-7, Published by IEEE, Sichuan, China, DOI 10.1109/FSKD.2012.6233733

[8] Kyeong B. L.; Jeong-hoon K.; Yong-San Y. \& Sang H. L. (2008). Obstacle-overcoming algorithm for unmanned ground vehicle with actively articulated suspensions on unstructured terrain, Proceedings of the International Conference on Control, Automation and Systems 2008, ISBN 978-89-950038-9-3, Published by IEEE, Seoul, South Korea, DOI 10.1109/ICCAS.2008.4694665

[9] Kyeong B. L.; Sukhoon P.; Suengwoo K.; Jae M. J. \& Yong-San Y. (2009). Behavior planning of an unmanned ground vehicle with actively articulated suspension to negotiate geometric obstacles, Proceedings of the 2009 IEEE/RSJ International Conference on Intelligent Robots and Systems, ISSN 2153-0858, ISBN 978-1-4244-38037, Published by IEEE, St. Louis, MO, USA, DOI 10.1109/IROS.2009.5354137

[10] Pierre L.; Ambroise K.; Michel L. \& Roland S. (2004). Wheel torque control for a rough terrain rover, Proceedings of the 2004 IEEE international Conference on Robotics and Automation, ISBN 0-7803-8232-3, Published by IEEE, New Orleans, LA, USA, DOI 10.1109/ROBOT.2004.1302456

[11] Leah K., Kurt T.; Patrick L. \& Garret C. (2015). Tip-over prevention: adaptive control development and experimentation, Proceedings of the 2015 IEEE International Conference on Robotics and Automation (ICRA), ISSN 1050-4729, ISBN 978-1-4799-6923-4, Published by IEEE, Seattle, WA, USA, DOI 10.1109/ICRA.2015.7139802

[12] Anastasios I. M.; Nikolas T.; Stergios L. R.; Daniel M. H. \& Larry M. (2007). Autonomous stair climbing for tracked vehicles. The International Journal of Robotics Research, Vol. 26, No. 7, (July 2007) pp. 737-758, DOI $10.1177 / 0278364907080423$

[13] Mihankhah E.; Kalantari A.; Aboosaeedan E.; Taghirad H.D. \& Moosavian S.Ali.A. (2009). Autonomous staircase detection and stair climbing for a tracked mobile robot using fuzzy controller, Proceedings of the 2008 IEEE International Conference on Robotics and Biomimetics, ISBN 978-1-4244-2678-2, Published by IEEE, Bangkok, Thailand, DOI 10.1109/ROBIO.2009.4913304

[14] Martin P. \& Karel Z.(2017). Controlling robot morphology from incomplete measurements. IEEE Transactions on Industrial Electronics, Vol. 64, No. 2, (Feb. 2017) pp. 1773-1782, ISSN 0278-0046

[15] Wenzeng G.; Shigong J.; Chengguo Z. \& Xueshan G. (2014). Development of a transformable wheel-track mobile robot and obstacle-crossing mode selection, Proceedings of the 2014 IEEE International Conference on Mechatronics and Automation, ISSN 2152-7431, ISBN 978-1-4799-3979-4, Published by IEEE, Tianjin, China, DOI 10.1109/ICMA.2014.6885957

[16] Michael B.; Bernd B. \& Dirk S. (2013). Hierarchical rough terrain motion planning using an optimal sampling-based method, Proceedings of the 2013 IEEE International Conference on Robotics and Automation (ICRA), ISSN 10504729, ISBN 978-1-4673-5643-5, Published by IEEE, Karlsruhe, Germany, DOI 10.1109/ICRA.2013.6631372 